\title{
High-accuracy waveforms for binary black hole inspiral, merger, and ringdown
}

\author{
Mark A. Scheel, ${ }^{1}$ Michael Boyle, ${ }^{1}$ Tony Chu, ${ }^{1}$ Lawrence E. Kidder, ${ }^{2}$ Keith D. Matthews, ${ }^{1}$ and Harald P. Pfeiffer ${ }^{1}$ \\ ${ }^{1}$ Theoretical Astrophysics 130-33, California Institute of Technology, Pasadena, California 91125, USA \\ ${ }^{2}$ Center for Radiophysics and Space Research, Cornell University, Ithaca, New York 14853, USA
}

(Received 9 October 2008; published 7 January 2009)

\begin{abstract}
The first spectral numerical simulations of 16 orbits, merger, and ringdown of an equal-mass nonspinning binary black hole system are presented. Gravitational waveforms from these simulations have accumulated numerical phase errors through ringdown of $\leqslant 0.1$ radian when measured from the beginning of the simulation, and $\lesssim 0.02$ radian when waveforms are time and phase shifted to agree at the peak amplitude. The waveform seen by an observer at infinity is determined from waveforms computed at finite radii by an extrapolation process accurate to $\lesssim 0.01$ radian in phase. The phase difference between this waveform at infinity and the waveform measured at a finite radius of $r=100 \mathrm{M}$ is about half a radian. The ratio of final mass to initial mass is $M_{f} / M=0.95162 \pm 0.00002$, and the final black hole spin is $S_{f} / M_{f}^{2}=0.68646 \pm 0.00004$.

DOI: 10.1103/PhysRevD.79.024003

PACS numbers: 04.25.D-, 04.25.dg, 04.30.- w, 04.30.Db
\end{abstract}

\section{INTRODUCTION}

Beginning with the groundbreaking binary black hole evolutions of Pretorius [1] and the development of the moving puncture method [2,3], it has recently become possible to solve Einstein's equations numerically for the inspiral, merger, and ringdown of two black holes in a binary orbit. Already these simulations have provided tests of post-Newtonian approximations [4-14], have allowed initial exploration of the orbital dynamics of spinning binaries [15-20], have determined the recoil velocity of the final black hole when the masses are unequal [21-24], and have led to the discovery of dramatically large recoil velocity from certain spin configurations [18,25-37].

Waveforms from these numerical simulations are important for gravitational-wave detectors such as LIGO and LISA. This is not only because detected waveforms can be compared with numerical models to measure astrophysical properties of the sources of gravitational radiation, but also because the detection probability itself can be increased via the technique of matched filtering [38], in which noisy data are convolved with numerical templates to enhance the signal.

However, binary black hole simulations are time consuming: a single simulation following approximately 10 orbits, merger, and ringdown typically requires a few weeks of runtime on approximately 50 or 100 processors of a parallel supercomputer, and typically such a simulation produces waveforms of only modest accuracy. This large computational expense precludes, for example, producing a full template bank of numerical waveforms covering the entire parameter space of black hole masses and spins. Hence, there has been much interest in construction of phenomenological analytical waveforms [7,39-41] that can be computed quickly and are calibrated by a small number of numerical simulations. While the accuracy of typical simulations is sufficient for creating LIGO detec- tion templates, it is most likely inadequate for LIGO parameter estimation and is far from what is required for LISA data analysis [42].

One approach to increasing the accuracy and efficiency of simulations is to adopt more efficient numerical methods. In particular, a class of numerical techniques known as spectral methods holds much promise. For smooth solutions, the errors produced by spectral methods decrease exponentially as computational resources are increased, whereas the errors of finite difference methods, the methods used by the majority of binary black hole simulations, decrease polynomially. Indeed, spectral methods have been used to produce very accurate initial data for binary black holes and neutron stars [43-56], and they have been used to produce the longest and most accurate binary black hole inspiral simulation to date $[9,57]$.

However, a key difficulty with time-dependent spectral binary black hole simulations has been handling the merger of the two holes. For example, the spectral simulations described in $[9,12,57]$ are very accurate and efficient, but they follow only the inspiral of the two black holes, and fail just before the holes merge. This is sufficient for some applications, such as comparing post-Newtonian formulas with numerical results during the inspiral and finding accurate analytic templates that match the numerical inspiral waveforms $[9,12]$, but for most purposes the merger is the most crucial part of the process: for instance the gravitational-wave emission is the strongest during merger, and details of the merger determine the recoil velocity of the final black hole.

In this paper we present a spectral binary black hole simulation that follows 16 orbits of the binary plus merger and ringdown of the merged black hole. In Sec. II we describe the equations, gauge conditions, and numerical methods we use to solve Einstein's equations; in particular, Secs. II C and II D describe changes to our gauge conditions that allow simulation of the merger, and our method 
for extending the evolution through ringdown. In Sec. III we discuss extraction of the gravitational waveform from the simulation, including the process of extrapolating the waveform to infinity. Section III also includes an estimate of the uncertainty in the waveform from several sources. Finally, in Sec. IV we discuss outstanding difficulties and future improvements.

\section{SOLUTION OF EINSTEIN'S EQUATIONS}

\section{A. Initial data}

The initial data describe two nonspinning black holes, each with Christodoulou mass $M / 2$, in quasicircular orbit with low eccentricity. The initial data are exactly as described in Ref. [9]. Briefly, initial data are constructed within the conformal thin sandwich formalism [58,59] using a pseudospectral elliptic solver [49]. We employ quasiequilibrium boundary conditions $[50,60]$ on spherical excision boundaries, choose conformal flatness and maximal slicing, and use Eq. (33a) of Ref. [53] as the lapse boundary condition. The spins of the black holes are made very small $\left(\sim 10^{-7}\right)$ via an appropriate choice of the tangential shift at the excision surfaces, as described in [53]. Finally, the initial orbital eccentricity is tuned to a very small value $\left(\sim 5 \times 10^{-5}\right)$ using the iterative procedure described in Ref. [9], which is an improved version of the procedure of Ref. [61].

\section{B. Evolution of the inspiral phase}

The evolution of the first $\sim 15$ binary orbits is identical to the simulation presented in Ref. [9]. We describe it here briefly in order to facilitate the presentation of our method for continuing the evolution through merger and ringdown, which is described in Secs. II C and II D.

The Einstein evolution equations are solved with the pseudospectral evolution code described in Ref. [57]. This code evolves a first-order representation [62] of the generalized harmonic system [63-65]. We handle the singularities by excising the black hole interiors from the computational domain. Our outer boundary conditions $[62,66,67]$ are designed to prevent the influx of unphysical constraint violations [68-74] and undesired incoming gravitational radiation $[75,76]$, while allowing the outgoing gravitational radiation to pass freely through the boundary.

We employ the dual-frame method described in Ref. [57]: we solve the equations in an "inertial frame" that is asymptotically Minkowski, but our domain decomposition is fixed in a "comoving frame" that rotates with respect to the inertial frame and also shrinks with respect to the inertial frame as the holes approach each other. The positions of the holes are fixed in the comoving frame; we account for the motion of the holes by dynamically adjusting the coordinate mapping between the two frames. Note that the comoving frame is referenced only internally in the code as a means of treating moving holes with a fixed domain. Therefore all coordinate quantities (e.g. black hole trajectories, wave-extraction radii) mentioned in this paper are inertial-frame values unless explicitly stated otherwise.

As described in [9], the mapping between inertial and comoving coordinates for the inspiral, expressed in polar coordinates relative to the center of mass of the system, is

$$
\begin{gathered}
r=\left[a(t)+(1-a(t)) \frac{r^{\prime 2}}{R_{0}^{\prime 2}}\right] r^{\prime}, \\
\theta=\theta^{\prime}, \\
\phi=\phi^{\prime}+b(t),
\end{gathered}
$$

where $a(t)$ and $b(t)$ are functions of time, and $R_{0}^{\prime}$ is a constant usually chosen to be roughly the radius of the outer boundary in comoving coordinates. Here primes denote the comoving coordinates. For the choice $R_{0}^{\prime}=$ $\infty$, the mapping is simply a rotation by $b(t)$ plus an overall contraction given by $a(t)$. The functions $a(t)$ and $b(t)$ are determined by a dynamical control system as described in Ref. [57]. This control system dynamically adjusts $a(t)$ and $b(t)$ so that the centers of the apparent horizons remain stationary in the comoving frame. Note that the outer boundary of the computational domain is at a fixed comoving radius $R_{\max }^{\prime}$, so the inertial-coordinate radius of the outer boundary $R_{\max }(t)$ is a function of time.

The gauge freedom in the generalized harmonic system is fixed via a freely specifiable gauge source function $H_{a}$ that satisfies the constraint

$$
0=\mathcal{C}_{a} \equiv \Gamma_{a b}{ }^{b}+H_{a},
$$

where $\Gamma_{b c}^{a}$ are the spacetime Christoffel symbols. To choose this gauge source function, we first define a new quantity $\tilde{H}_{a}$ that has the following two properties: (1) $\tilde{H}_{a}$ transforms like a tensor, and (2) in inertial coordinates $\tilde{H}_{a}=H_{a}$. We choose $H_{a}$ so that the constraint equation (4) is satisfied initially, and we demand that $\tilde{H}_{a^{\prime}}$ is constant in the moving frame, i.e., that $\partial_{t^{\prime}} \tilde{H}_{a^{\prime}}=0$.

\section{Extending inspiral runs through merger}

If the inspiral runs described above are allowed to continue without any modification of the algorithm, then as the binary approaches merger, the horizons of the black holes become extremely distorted and the dynamical fields begin to develop sharp (but numerically convergent) features near each hole. These features grow rapidly in time, eventually halting the simulation before merger. This is due to a gauge effect: The gauge condition used during the inspiral, namely fixing $H_{a}$ in time in the comoving frame, was chosen based on the idea that each black hole is in quasiequilibrium in this frame. Once the black holes begin to interact strongly, this gauge condition no longer allows the coordinates to sufficiently react to the changing geometry, and coordinate singularities develop. 
Therefore we must modify our gauge conditions in order to handle merger. Because the inspiral gauge works so well before merger, we choose to remain in that gauge until some time $t=t_{g}$, and then we change (smoothly) to a new gauge.

We have experimented with several gauge conditions [77], but so far the simplest gauge choice that works, and the one used in the simulations presented here, is based on the gauge treatment of Pretorius [1,65,78]: We promote the gauge source function $H_{a}$ to an independent dynamical field that satisfies

$$
\nabla^{c} \nabla_{c} H_{a}=Q_{a}\left(x, t, \psi_{a b}\right)+\xi_{2} t^{b} \partial_{b} H_{a},
$$

where $\nabla^{c} \nabla_{c}$ is the curved space scalar wave operator (i.e. each component of $H_{a}$ is evolved as a scalar), $\psi_{a b}$ is the spacetime metric, and $t^{a}$ is the timelike unit normal to the hypersurface. The driving function $Q_{a}$ is

$$
\begin{gathered}
Q_{t}=f(x, t) \xi_{1} \frac{1-N}{N^{\eta}}, \\
Q_{i}=g(x, t) \xi_{3} \frac{N_{i}}{N^{2}} .
\end{gathered}
$$

Here $N$ and $N^{i}$ are the lapse function and the shift vector, $\eta, \xi_{1}, \xi_{2}$, and $\xi_{3}$ are constants, and $f(x, t)$ and $g(x, t)$ are prescribed functions of the spacetime coordinates (we describe our choices for these objects below).

Equation (5) is a damped, driven wave equation with damping parameter $\xi_{2}$ and driving function $Q_{a}$. The driving term $Q_{t}$ in Eq. (6) was introduced by Pretorius $[1,65]$ to drive the lapse function towards unity so as to prevent it from becoming small. The driving term $Q_{i}$ is new; it drives the shift vector towards zero near the horizons. This causes the horizons to expand in coordinate space, and has the effect of smoothing out the dynamical fields near the horizon and preventing gauge singularities from developing. A different gauge choice that causes similar coordinate expansion of the horizons was introduced in Ref. [79]. Care must be taken so that the horizons do not expand too quickly relative to the excision boundaries; otherwise the characteristic fields will fail to be purely outgoing (into the holes) at the excision boundaries, and excision will fail. We find that with appropriate choices of $\xi_{1}, \xi_{3}, f(x, t)$, and $g(x, t)$ as described below, the horizons expand gradually and not too rapidly.

For the runs presented here we choose $\eta=4, \xi_{1}=0.1$, $\xi_{2}=10$, and $\xi_{3}=0.4$. The functions $f(x, t)$ and $g(x, t)$ in Eqs. (6) and (7) are chosen based on two criteria: the first is that the driving terms $Q_{a}$ are nonzero only near the black holes where they are needed; if these terms are nonzero in the wave-extraction zone they lead to complicated gauge dynamics in this region, making waveform extraction difficult. The second criterion is that the driving terms are turned on in a gradual manner so that the gauge does not change too rapidly. We choose

$$
\begin{aligned}
f(x, t) & =g(x, t) \\
& =\left(2-e^{-\left(t-t_{g}\right) / \sigma_{1}}\right)\left(1-e^{-\left(t-t_{g}\right)^{2} / \sigma_{2}^{2}}\right) e^{-r^{\prime 2} / \sigma_{3}^{2}},
\end{aligned}
$$

where $r^{\prime}$ is the coordinate radius in comoving coordinates, and the constants are $\sigma_{1} \sim 17.5 M, \sigma_{2} \sim 15 M$, and $\sigma_{3} \sim$ $40 M$. Here $M$ is the sum of the initial Christodoulou masses of the two holes.

Equation (5) is a second-order hyperbolic equation, which we evolve in first-order form by defining new fields $\Pi_{a}^{H}$ and $\Phi_{i a}^{H}$, representing (up to the addition of constraints) the appropriate time and space derivatives of $H_{a}$, respectively:

$$
\begin{gathered}
\Pi_{a}^{H}=-t^{b} \partial_{b} H_{a}, \\
\Phi_{i a}^{H}=\partial_{i} H_{a} .
\end{gathered}
$$

The representation of wave equations of this type in firstorder form is well understood, see e.g., Refs. [62,80]; the result for Eq. (5) is

$$
\begin{gathered}
\partial_{t} H_{a}=-N \Pi_{a}^{H}+N^{k} \Phi_{k a}^{H}, \\
\partial_{t} \Pi_{a}^{H}=N^{k} \partial_{k} \Pi_{a}^{H}-N g^{k i} \partial_{k} \Phi_{i a}^{H}-\gamma_{2}^{H} N^{k} \partial_{k} H_{a} \\
+\gamma_{2}^{H} N^{k} \Phi_{k a}^{H}+N\left(\Gamma^{k j}{ }_{j}-g^{k j} \partial_{j} N\right) \Phi_{k a}^{H} \\
+N K \Pi_{a}^{H}+Q_{a},
\end{gathered}
$$

$$
\begin{aligned}
\partial_{t} \Phi_{i a}^{H}= & N^{k} \partial_{k} \Phi_{i a}^{H}-N \partial_{i} \Pi_{a}^{H}+\gamma_{2}^{H} N \partial_{i} H_{a}-\Pi_{a}^{H} \partial_{i} N \\
& +\Phi_{k a}^{H} \partial_{i} N^{k}-\gamma_{2}^{H} N \Phi_{i a}^{H},
\end{aligned}
$$

where $g_{i j}$ is the spatial metric and $K$ is the trace of the extrinsic curvature. We choose the constraint-damping parameter $\gamma_{2}^{H}$ to be $\gamma_{2}^{H}=4 / M$.

These equations are symmetric hyperbolic, and require boundary conditions on all incoming characteristic fields at all boundaries. The characteristic fields for Eqs. (11)-(13) in the direction of a unit spacelike covector $n_{i}$ are

$$
\begin{gathered}
U_{a}^{H \pm}=\Pi_{a}^{H} \pm n^{i} \Phi_{i a}^{H}-\gamma_{2}^{H} H_{a}, \\
Z_{a}^{H 1}=H_{a}, \\
Z_{i a}^{H 2}=\left(\delta_{i}^{k}-n_{i} n^{k}\right) \Phi_{k a}^{H} .
\end{gathered}
$$

The (coordinate) characteristic speeds for $U_{a}^{H \pm}, Z_{a}^{H 1}$, and $Z_{i a}^{H 2}$ are $\pm N-n_{i} N^{i}, 0$, and $-n_{i} N^{i}$, respectively.

At the excision boundaries all characteristic fields are outgoing (i.e. into the holes) or nonpropagating, so no boundary conditions are necessary and none are imposed. At the outer boundary, we must impose boundary conditions on $U_{a}^{H-}$ and $Z_{i a}^{H 2}$. Define

$$
\begin{gathered}
D_{t}\left(U_{a}^{H \pm}\right) \equiv \partial_{t} \Pi_{a}^{H} \pm n^{i} \partial_{t} \Phi_{i a}^{H}-\gamma_{2}^{H} \partial_{t} H_{a}, \\
D_{t}\left(Z_{a}^{H 1}\right) \equiv \partial_{t} H_{a},
\end{gathered}
$$




$$
D_{t}\left(Z_{i a}^{H 2}\right) \equiv\left(\delta_{i}^{k}-n_{i} n^{k}\right) \partial_{t} \Phi_{k a}^{H},
$$

where the time derivatives on the right-hand side are evaluated using Eqs. (11)-(13). Then we impose the following boundary conditions:

$$
\begin{gathered}
\partial_{t} U_{a}^{H-}=-\gamma_{2}^{H} D_{t}\left(Z_{a}^{H 1}\right), \\
\partial_{t} Z_{i a}^{H 2}=D_{t}\left(Z_{i a}^{H 2}\right)+2 n_{k} N^{k} n^{j} \partial_{[i} \Phi_{j] a}^{H} .
\end{gathered}
$$

Equation (20) is the outgoing-wave boundary condition described in detail in Ref. [80]. Equation (21) ensures that violations of the artificial constraint $C_{i a} \equiv$ $\Phi_{i a}^{H}-\partial_{i} H_{a}=0$ do not enter the domain through the boundary; it is the direct analogue of the constraintpreserving boundary condition we apply to the analogous variable in the generalized harmonic formulation of Einstein's equations, Eq. (65) of Ref. [62].

Note that Eqs. (11)-(13) involve only first derivatives of the spacetime metric, and similarly, the generalized harmonic Einstein equations involve only first derivatives of $H_{a}$. Therefore, adding Eqs. (11)-(13) to the system does not change the hyperbolicity or characteristic fields of the generalized harmonic Einstein equations, so we can impose the same boundary conditions on the generalized harmonic variables as we do during the inspiral, as described in Refs. [57,66].

Equations (11)-(13) require as initial data the values of $H_{a}$ and $\Pi_{a}^{H}$ at $t=t_{g}$. These quantities can be computed from the gauge choice used during the inspiral for $t \leq t_{g}$, so we choose them to be continuous at $t=t_{g}$.

Note that Eqs. (11)-(13) and the boundary conditions (20) and (21) are written in the inertial-coordinate system. The equations are actually solved in the comovingcoordinate system using the dual-frame method described in Ref. [57].

With the modifications to the gauge conditions described here, the evolution of the binary can be tracked up until (and shortly after) the formation of a common horizon that encompasses both black holes. Because of the more rapid dynamics and the distortions of the horizons during the merger, we typically increase the numerical resolution slightly when we make these changes to the gauge con- ditions (this is the difference between the first and second entry in the $N_{\text {pts }}$ column in Table I). After the common horizon forms, the problem reduces to evolving a single highly distorted dynamical black hole, rather than two separate black holes. We change the algorithm to take advantage of this, as described in the next section.

\section{Evolution from merger through ringdown}

We make three main changes to our evolution algorithm once we detect a common apparent horizon. First, because there is now only one black hole and not two, we interpolate all variables onto a new computational domain that contains only a single excised region. Second, we choose a new comoving-coordinate system (and a corresponding mapping to inertial coordinates) so that the new excision boundary tracks the shape of the (distorted, rotating, pulsating) apparent horizon in the inertial frame, and so that the outer boundary behaves smoothly in time. Third, we modify the gauge conditions so that the shift vector is no longer driven towards zero, allowing the solution to eventually relax to a time-independent state. We now discuss these three changes in detail.

Our new computational domain contains only a single excised region, and is much simpler than the one used until merger. It consists only of nested spherical-shell subdomains that extend from a new excision boundary $R_{\min }^{\prime \prime}$, chosen to be slightly inside the common apparent horizon, to an outer boundary $R_{\max }^{\prime \prime}$ that coincides with the outer boundary of the old domain.

To understand how we choose our new comoving frame, first recall that in the dual-frame technique [57], the comoving frame is the one in which the computational domain is fixed, the inertial frame is the one in which the coordinates are Minkowski-like at infinity, and the two frames are related by a mapping that is chosen so that the computational domain tracks the motion of the black holes. Let $x^{a}$ represent the inertial coordinates (which are the same before and after merger), let $x^{\prime a}$ represent the old comoving coordinates, and let $x^{\prime \prime a}$ represent the new comoving coordinates. The mapping between $x^{\prime a}$ and $x^{a}$ is given by Eqs. (1)-(3). The mapping between $x^{\prime \prime a}$ and $x^{a}$ is chosen to be

TABLE I. Outer boundary parameters, collocation points, and CPU usage for several zero-spin binary black hole evolutions. The first column identifies the inspiral run in the nomenclature of Ref. [9]. $N_{\mathrm{pts}}$ is the approximate number of collocation points used to cover the entire computational domain. The three values for $N_{\mathrm{pts}}$ are those for the inspiral, merger, and ringdown portions of the simulation, which are described in Sections II B, II C, and II D, respectively. The outer boundary parameters $R_{\max }^{\prime}, R_{\max }^{\prime \prime}$ and $R_{0}^{\prime}$, as well as run times $T$, are in units of the initial Christodoulou mass $M$ of the system, which provides a natural time and length scale.

\begin{tabular}{lccccrr}
\hline \hline Run & $R_{\max }^{\prime}$ & $R_{\max }^{\prime \prime}$ & $R_{0}^{\prime}$ & $N_{\mathrm{pts}}$ & CPU-h & CPU-h/T \\
\hline 30c1/N4 & 462 & 462 & 698 & $\left(57^{3}, 59^{3}, 57^{3}\right)$ & 8800 & 2.0 \\
30c1/N5 & 462 & 462 & 698 & $\left(62^{3}, 66^{3}, 63^{3}\right)$ & 15000 & 3.4 \\
30c1/N6 & 462 & 462 & 698 & $\left(67^{3}, 73^{3}, 70^{3}\right)$ & 23000 & 5.3 \\
30c2/N6 & 722 & 96 & $\infty$ & $\left(71^{3}, 76^{3}, 63^{3}\right)$ & 25000 & 5.7 \\
\hline \hline
\end{tabular}




$$
\begin{gathered}
r=\tilde{r}\left[1+\sin ^{2}\left(\pi \tilde{r} / 2 R_{\max }^{\prime \prime}\right)\left(A(t) \frac{R_{\max }^{\prime}}{R_{\max }^{\prime \prime}}+(1-A(t))\right.\right. \\
\left.\left.\times \frac{R_{\max }^{\prime 3}}{R_{\max }^{\prime \prime} R_{0}^{\prime 2}}-1\right)\right], \\
\tilde{r}=r^{\prime \prime}-q\left(r^{\prime \prime}\right) \sum_{\ell=0}^{\ell_{\max }} \sum_{m=-\ell}^{\ell} \lambda_{\ell m}(t) Y_{\ell m}\left(\theta^{\prime \prime}, \phi^{\prime \prime}\right), \\
\theta=\theta^{\prime \prime}, \\
\phi=\phi^{\prime \prime}+B(t),
\end{gathered}
$$

where $R_{\max }^{\prime}$ is the outer boundary of the premerger computational domain in the old comoving coordinates, and $q\left(r^{\prime \prime}\right), A(t), B(t)$, and $\lambda_{\ell m}(t)$ are functions we will now discuss.

First we describe the angular map: The function $B(t)$ is chosen so that the new comoving frame initially rotates with respect to the inertial frame, but this rotation slows to a halt after a short time. In particular,

$$
B(t)=B_{0}+\left(B_{1}+B_{2}\left(t-t_{m}\right)\right) e^{-\left(t-t_{m}\right) / \tau_{B}},
$$

where the constants $B_{0}, B_{1}$, and $B_{2}$ are chosen so that $B(t)$ matches smoothly onto $b(t)$ from Eq. (3): $B\left(t_{m}\right)=b\left(t_{m}\right)$, $\dot{B}\left(t_{m}\right)=\dot{b}\left(t_{m}\right)$, and $\ddot{B}\left(t_{m}\right)=\ddot{b}\left(t_{m}\right)$. Here $t_{m}$ is the time at which we transition to the new domain decomposition. The constant $\tau_{B}$ is chosen to be on the order of $20 M$.

The radial map is a composition of two individual maps: Eqs. (22) and (23). The purpose of Eq. (22) is to match the outer boundary of the new domain smoothly onto that of the old domain, while far from the outer boundary Eq. (22) approaches the identity. We have found that without the use of Eq. (22), the (inertial-coordinate) location of the boundary changes nonsmoothly at $t=t_{m}$, thereby generating a spurious ingoing gauge pulse that spoils waveform extraction. The function $A(t)$ is

$$
A(t)=A_{0}+\left(A_{1}+A_{2}\left(t-t_{m}\right)\right) e^{-\left(t-t_{m}\right) / \tau_{A}},
$$

where the constants $A_{0}, A_{1}$, and $A_{2}$ are chosen so that $A(t)$ matches smoothly onto $a(t)$ from Eq. (1): $A\left(t_{m}\right)=a\left(t_{m}\right)$, $\dot{A}\left(t_{m}\right)=\dot{a}\left(t_{m}\right)$, and $\ddot{A}\left(t_{m}\right)=\ddot{a}\left(t_{m}\right)$. The constant $\tau_{A}$ is chosen to be on the order of $5 M$.

The other piece of the radial map, Eq. (23), is chosen so that the apparent horizon is nearly spherical in the new comoving coordinates $x^{\prime \prime a}$. The function $q\left(r^{\prime \prime}\right)$ is

$$
q\left(r^{\prime \prime}\right)=e^{-\left(r^{\prime \prime}-R_{\mathrm{AH}}^{\prime \prime}\right)^{3} / \sigma_{q}^{3}},
$$

where $R_{\mathrm{AH}}^{\prime \prime}$ is the radius of the apparent horizon in comoving coordinates, and $\sigma_{q}$ is a constant of order 20M. This function $q\left(r^{\prime \prime}\right)$ ensures that the piece of the radial map represented by Eq. (23) acts only in the vicinity of the merged hole and not in the exterior wave-extraction region.

We now discuss the choice of the functions $\lambda_{\ell m}(t)$ that appear in Eq. (23). Given the known location of the appar- ent horizon in inertial coordinates, the $\lambda_{\ell m}(t)$ determine the shape of the apparent horizon in comoving coordinates. At $t=t_{m}$, we choose these quantities so that the apparent horizon is spherical (up to spherical harmonic component $\ell=\ell_{\max }$ ) in comoving coordinates: that is, if the comoving-coordinate radius of the apparent horizon as a function of angles is written as

$$
r_{\mathrm{AH}}^{\prime \prime}\left(\theta^{\prime \prime}, \phi^{\prime \prime}\right) \equiv \sum_{\ell=0}^{\ell_{\max }} \sum_{m=-\ell}^{\ell} Q_{\ell m}(t) Y_{\ell m}\left(\theta^{\prime \prime}, \phi^{\prime \prime}\right),
$$

then for $1 \leq \ell \leq \ell_{\max }$ we choose $\lambda_{\ell m}\left(t_{m}\right)$ so that $Q_{\ell m}\left(t_{m}\right)=0$. In addition, we choose $\lambda_{00}\left(t_{m}\right)=0$; this determines $R_{\mathrm{AH}}^{\prime \prime}$. For $t>t_{m}, \lambda_{\ell m}(t)$ are determined by a dynamical feedback control system identical to the one described in Ref. [57], which adjusts these functions so that the apparent horizon is driven to a sphere (up to spherical harmonic component $\ell=\ell_{\max }$ ) in comoving coordinates. This dynamical feedback control allows us to freely choose the first and second time derivatives of $\lambda_{\ell m}$ at $t=t_{m}$. Simply choosing these to be zero causes the control system to oscillate wildly before settling down, and unless the time step is very small, these oscillations are large enough that the excision boundary crosses the horizon and our excision algorithm fails. So instead, we obtain the time derivatives of $\lambda_{\ell m}$ by finding the apparent horizon at several times surrounding $t=t_{m}$, computing $\lambda_{\ell m}$ at these times, and finite differencing in time. For the equal-mass zero-spin merger presented here, in Eq. (23) it suffices to sum only over even $\ell$ and $m$ and to choose $\ell_{\max }=6$.

The last change we make before continuing the simulation past merger is to modify the functions $f(x, t)$ and $g(x, t)$, which before merger were given by Eq. (8), to

$$
\begin{gathered}
f(x, t)=\left(2-e^{-\left(t-t_{g}\right) / \sigma_{1}}\right)\left(1-e^{-\left(t-t_{g}\right)^{2} / \sigma_{2}^{2}}\right) e^{-r^{\prime \prime 2} / \sigma_{3}^{2},} \\
g(x, t)=f(x, t) e^{-\left(t-t_{m}\right)^{2} / \sigma_{4}^{2},}
\end{gathered}
$$

where $\sigma_{4}=7 M$. The modification of $g(x, t)$ turns off the term in the gauge evolution equations that drives the shift to zero near the holes. Before merger, it is advantageous to have the shift driven to zero so that the horizons expand in coordinate space and so that growing gauge modes remain inside the common horizon. After merger, however, it is no longer desirable for the horizon to expand, since this would prevent the solution from eventually settling down to a time-independent state in which the horizon is stationary with respect to the coordinates.

To summarize, the steps involved in the transition from evolving a binary black hole spacetime to evolving a merged single black hole spacetime are as follows: (1) Find the common apparent horizon in the inertial frame at several times near $t=t_{m}$. (2) Solve for the $\lambda_{\ell m}\left(t_{m}\right)$ that make the horizon spherical in the comoving frame, and simultaneously solve for $R_{\mathrm{AH}}^{\prime \prime}$. (3) Choose the inner boundary of the new computational domain $R_{\min }^{\prime \prime}$ to be slightly 
less than $R_{\mathrm{AH}}^{\prime \prime}$, and choose the outer boundary $R_{\max }^{\prime \prime}$ [for sufficiently small $a\left(t_{m}\right)$ it is necessary to choose $R_{\max }^{\prime \prime}<$ $R_{\max }^{\prime}$ so that the mapping (22) is invertible]. At this point the computational domain and the mapping (22)-(25) have been determined. (4) Interpolate all dynamical variables from the old computational domain onto the new one. This interpolation is done via the spectral expansion in the old domain, so it introduces no additional error. (5) Modify the gauge source evolution equations so that the shift is no longer driven to zero. (6) Continue the evolution on the new computational domain. All of these steps can be automated.

\section{E. Properties of the numerical solution}

In Table I we list outer boundary parameters, resolutions, and run times of several runs we have done using the algorithm described above. Three of these runs are identical except for numerical resolution, and the fourth is performed on a different domain with a different outer boundary location. As discussed above, the outer boundary of our simulation varies in time because of the dual-frame approach we use to follow the black holes. Figure 1 is a spacetime diagram illustrating the region of spacetime being evolved in our simulation.

We do not explicitly enforce either the Einstein constraints or the secondary constraints that arise from writing the system in first-order form. Therefore, examining how well these constraints are satisfied provides a useful consistency check. Figure 2 shows the constraint violations for run $30 \mathrm{c} 1$. The top panel shows the $L^{2}$ norm of all the constraint fields of our first-order generalized harmonic system, normalized by the $L^{2}$ norm of the spatial gradients
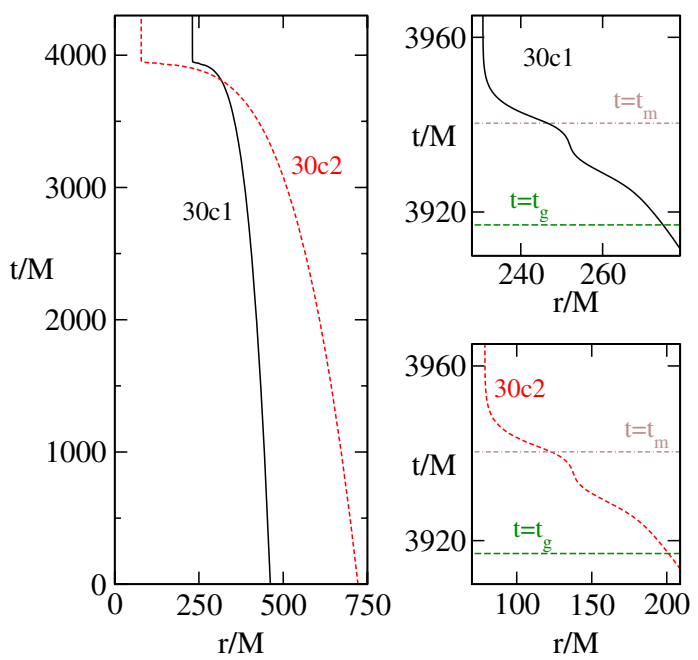

FIG. 1 (color online). Spacetime diagram showing the spacetime volume simulated by the numerical evolutions listed in Table I. Each curve represents the worldline of the outer boundary for a particular simulation. The magnified views on the right show that the outer boundary moves smoothly near merger. The transition times $t_{g}=3917 M$ and $t_{m}=3940 M$ are indicated on the right panels.

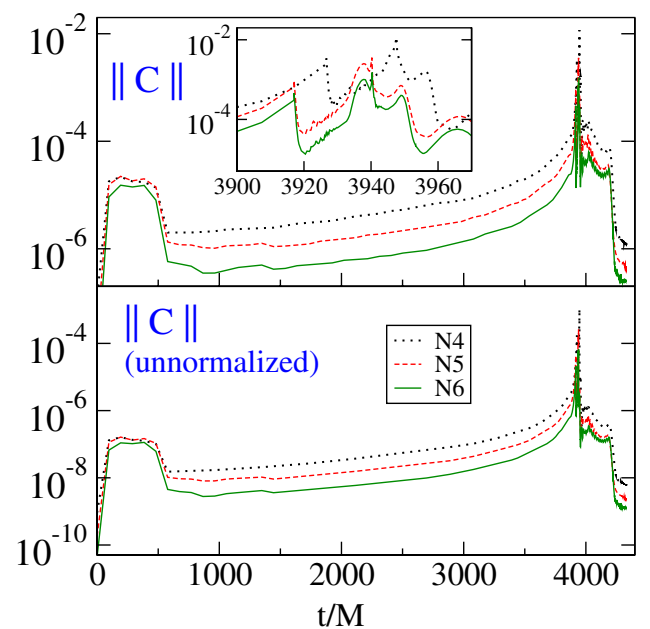

FIG. 2 (color online). Constraint violations of run 30c1. The top panel shows the $L^{2}$ norm of all constraints, normalized by the $L^{2}$ norm of the spatial gradients of all dynamical fields. The bottom panel shows the same data, but without the normalization factor. The $L^{2}$ norms are taken over the portion of the computational volume that lies outside apparent horizons.

of the dynamical fields [see Eq. (71) of Ref. [62]]. The bottom panel shows the same quantity, but without the normalization factor [i.e., just the numerator of Eq. (71) of Ref. [62] ]. The $L^{2}$ norms are taken over the portion of the computational volume that lies outside apparent horizons. At early times, $t<500 M$, the constraints converge rather slowly with resolution because the junk radiation contains high frequencies. Convergence is more rapid during the smooth inspiral phase, after the junk radiation has exited through the outer boundary.

The constraints increase as the holes approach each other and the solution becomes increasingly distorted. At $t=3917 M(t=3927 M$ for resolution $\mathrm{N} 4)$, the gauge conditions are changed (cf. Sec. IIC) and the resolution is increased slightly (compare the first and second entry in the $N_{\text {pts }}$ column in Table I). Because of the change of resolution, the constraints drop rapidly by almost 2 orders of magnitude, but then they begin to grow again. The transition to a single-hole evolution (cf. Sec. II D) occurs at $t=3940 M(t=3948 M$ for resolution N4). At this time the constraint norm drops by about an order of magnitude because the region in which the largest constraint violations occur-the interior of the common horizon-is newly excised.

After the binary proceeds through inspiral, merger, and ringdown, it settles down to a final stationary black hole. In our simulation this final state is not expressed in any standard coordinate system used to describe Kerr spacetime, but nevertheless the final mass and spin of the hole can be determined. The area $A$ of the apparent horizon provides the irreducible mass of the final black hole,

$$
M_{\mathrm{irr}}=\sqrt{A / 16 \pi}
$$


TABLE II. Physical parameters describing the equal-mass nonspinning binary black hole evolutions presented here. The dimensionful quantity $M$ is the initial sum of the Christodoulou masses of the black holes. Uncertainty estimates include numerical uncertainties and the effects of varying the outer boundary location.

Initial orbital eccentricity:

Initial spin of each hole:

Time of evolution:

Final Christodoulou mass:

Final spin:

which we find to be $M_{\mathrm{irr}} / M=0.88433 \pm 0.00001$, where $M$ is the sum of the initial irreducible masses of the black holes. The uncertainty in $M_{\text {irr }} / M$ is determined from the difference between runs 30c1/N6, 30c1/N5, and 30c2/N6, so it includes only uncertainties due to numerical resolution and outer boundary location. We have verified that the uncertainty due to the finite resolution of our apparent horizon finder is negligible.

The final spin $S_{f}$ of the black hole can be computed by integrating a quasilocal angular momentum density over the final apparent horizon $[81,82]$. Our implementation of this method is described in detail in Appendix A of [55]. Furthermore, an alternative method of computing the final spin, which is based on evaluating the extremal values of the two-dimensional scalar curvature on the apparent horizon and comparing these values to those obtained analytically for a Kerr black hole, is also described in [55]. Using these measures, we determine the dimensionless spin of the final black hole to be $S_{f} / M_{f}^{2}=0.68646 \pm 0.00004$, where the uncertainty is dominated by the difference between runs 30c1/N6 and 30c1/N5 rather than by the differences between different methods of measuring the spin. Here $M_{f}$ is the Christodoulou mass of the final black hole,

$$
M_{f}^{2}=M_{\mathrm{irr}}^{2}+\frac{S_{f}^{2}}{4 M_{\mathrm{irr}}^{2}} .
$$

We find that the ratio of the final to initial black hole mass is $M_{f} / M=0.95162 \pm 0.00002$. The mass and spin of the final hole are consistent with those found by other groups [2,4,83-85]. Physical parameters describing the evolutions are summarized in Table II.

\section{COMPUTATION OF THE WAVEFORM}

The numerical solution of Einstein's equations obtained using the methods described above yields the spacetime metric and its first derivatives at all points in the computational domain. In this section we describe how this solution is used to compute the key quantity relevant for gravitational-wave observations: the gravitational waveform as seen by an observer infinitely far from the source.

\section{A. Waveform extraction}

Gravitational waves are extracted from the simulation on a sphere of coordinate radius $r$ using the NewmanPenrose scalar $\Psi_{4}$, following the same procedure as in Refs. [61,86]. To summarize, we compute

$$
\Psi_{4}=-C_{\alpha \mu \beta \nu} \ell^{\mu} \ell^{\nu} \bar{m}^{\alpha} \bar{m}^{\beta}
$$

where

$$
\begin{aligned}
\ell^{\mu} & =\frac{1}{\sqrt{2}}\left(t^{\mu}-r^{\mu}\right), \\
m^{\mu} & =\frac{1}{\sqrt{2} r}\left(\frac{\partial}{\partial \theta}+i \frac{1}{\sin \theta} \frac{\partial}{\partial \phi}\right)^{\mu} .
\end{aligned}
$$

Here $(r, \theta, \phi)$ denote the standard spherical coordinates in the inertial frame, $t^{\mu}$ is the timelike unit normal to the spatial hypersurface, and $r^{\mu}$ is the outward-pointing unit normal to the extraction sphere. We then expand $\Psi_{4}$ in terms of spin-weighted spherical harmonics of weight -2 :

$$
\Psi_{4}(t, r, \theta, \phi)=\sum_{l m} \Psi_{4}^{l m}(t, r)_{-2} Y_{l m}(\theta, \phi),
$$

where the $\Psi_{4}^{l m}$ are expansion coefficients defined by this equation.

Note that our choice of $m^{\mu}$ is not exactly null nor exactly of unit magnitude at finite $r$, as is required by the standard definition. The resulting $\Psi_{4}^{l m}$ computed at finite $r$ will therefore disagree with the waveforms observed at infinity. Our definition does, however, agree with the standard definition of $\Psi_{4}^{l m}$ as $r \rightarrow \infty$. Because we extrapolate the extracted waves to find the asymptotic radiation field (see Sec. III C), these tetrad effects should not play a role: Relative errors in $\Psi_{4}^{l m}$ introduced by using the simple coordinate tetrad fall off like powers of $M / r$, and thus should vanish after extrapolating to obtain the asymptotic behavior. More careful treatment of the extraction method-such as those discussed in Refs. [87-89]-may improve the quality of extrapolation and would be interesting to explore in the future.

In this paper, we focus on the dominant $(l, m)=(2,2)$ mode. Following common practice (see e.g. [84,85]), we split the extracted waveform into real phase $\phi$ and real amplitude $A$, defined by

$$
\Psi_{4}^{22}(r, t)=A(r, t) e^{-i \phi(r, t)} .
$$

The gravitational-wave frequency is given by

$$
\omega=\frac{d \phi}{d t} .
$$

The minus sign in Eq. (37) is chosen so that the phase increases in time and $\omega$ is positive.

The $(l, m)=(2,2)$ waveform, extracted at a single radius for run 30c1/N6, is shown in Fig. 3. The short pulse at $t \sim 200 M$ is caused by imperfect initial data that are not 

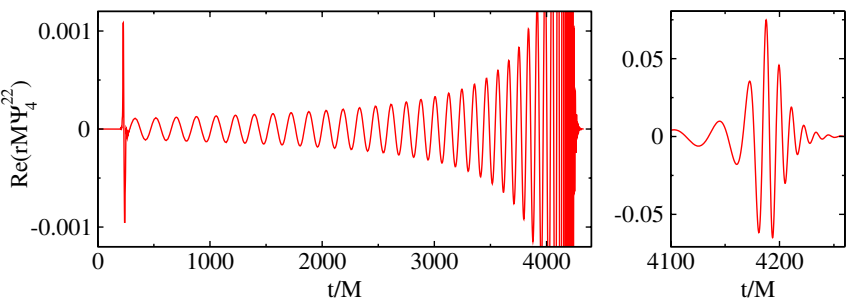

FIG. 3 (color online). Gravitational waveform extracted at finite radius $r=225 M$, for the case $30 \mathrm{c} 1 / \mathrm{N} 6$ in Table I. The left panel zooms in on the inspiral waveform, and the right panel zooms in on the merger and ringdown.

precisely in equilibrium; this pulse is usually referred to as "junk radiation."

\section{B. Convergence of extracted waveforms}

In this section we examine the convergence of the gravitational waveforms extracted at fixed radius, without extrapolation to infinity. This allows us to study the behavior of our code without the complications of extrapolation. The extrapolation process and the resulting extrapolated waveforms are discussed in Sec. III C.

Figure 4 shows the convergence of the gravitationalwave phase $\phi$ and amplitude $A$ with numerical resolution. For this plot, the waveform was extracted at a fixed inertialcoordinate radius of $r=60 \mathrm{M}$. This fairly small extraction radius was chosen to allow a comparison of the simulations $30 \mathrm{c} 1$ and 30c2. Each solid line in the top panel shows the absolute difference between $\phi$ computed at some particular resolution and $\phi$ computed from our highest-resolution

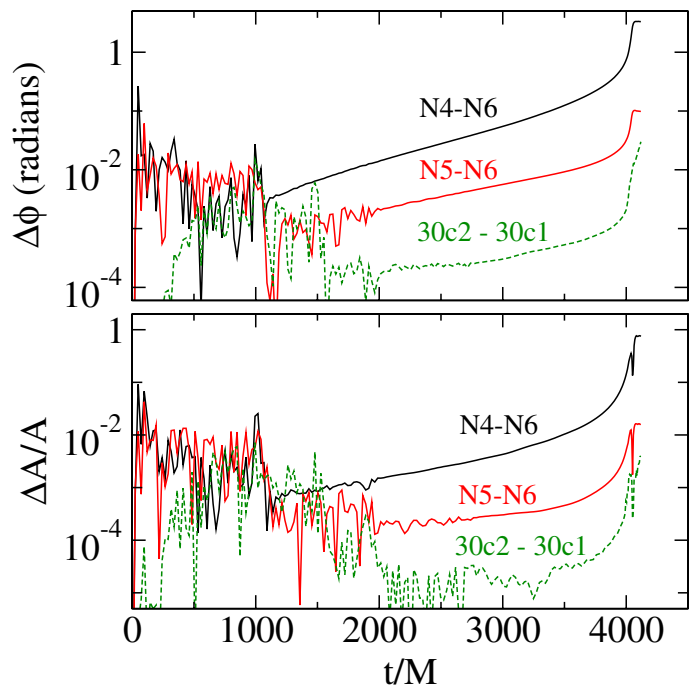

FIG. 4 (color online). Convergence of waveforms with numerical resolution and outer boundary location. Shown are phase and amplitude differences between numerical waveforms $\Psi_{4}^{22}$ computed using different numerical resolutions. Shown also is the difference between our highest-resolution waveforms using two different outer boundary locations. All waveforms are extracted at $r=60 \mathrm{M}$, and no time shifting or phase shifting is done to align waveforms.

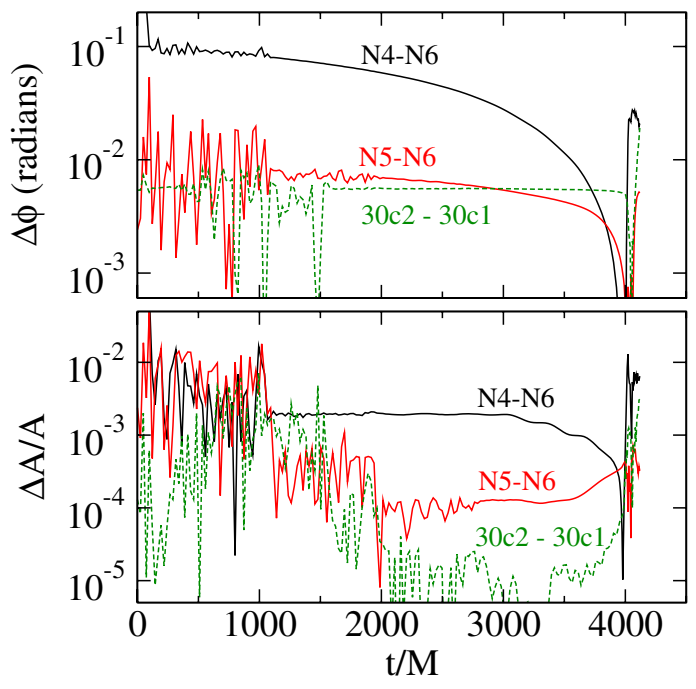

FIG. 5 (color online). Convergence of waveforms with numerical resolution and outer boundary location. Same as Fig. 4 except waveforms are time shifted and phase shifted so that the maximum amplitude occurs at the same time and phase.

run, labeled 30c1/N6 in Table I. The solid curves in the bottom panel similarly show the relative amplitude differences. When subtracting results at different resolutions, no time or phase adjustment has been performed. The noise at early times is due to "junk radiation" generated near $t=$ 0 . While most of this radiation leaves through the outer boundary after one crossing time, some remains visible for a few crossing times. ${ }^{1}$ The plots show that the phase difference accumulated over 16 orbits plus merger and ringdown is less than 0.1 radians for our medium resolution, and the relative amplitude differences are less than 0.015 ; these numbers can be taken as an estimate of the numerical truncation error of our medium resolution run.

Also shown as a dotted curve in each panel of Fig. 4 is the difference between our highest-resolution run, 30c1/ N6, and a similar run but with a different outer boundary location, 30c2/N6. The 30c2 run initially has a more distant outer boundary than $30 \mathrm{c} 1$, but during the inspiral the outer boundary moves rapidly inward, as seen in Fig. 1, so that extraction of the full waveform is possible only for extraction radii $r \lessgtr 75 M$. Comparing runs $30 \mathrm{c} 1$ and $30 \mathrm{c} 2$ provides an estimate of the uncertainty in the waveform due to outer boundary effects such as imperfect boundary conditions that might reflect outgoing waves. From Fig. 4 we estimate this uncertainty to be 0.03 radians in phase and half a percent in amplitude (when no time shift is applied).

Figure 5 is the same as Fig. 4 except each waveform is time shifted and phase shifted so that the maximum amplitude of the wave occurs at the same time and phase. This

\footnotetext{
${ }^{1}$ The junk radiation at early times is discussed in more detail in Ref. [9] [specifically, just before Eq. (9) and in the third paragraph of Sec. IIE], which presents the exact same waveform as shown here but without merger and ringdown.
} 
type of comparison is relevant for analysis of data from gravitational-wave detectors: when comparing experimental data with numerical detection templates, the template will be shifted in both time and phase to best match the data. For this type of comparison, Fig. 5 shows that the numerical truncation error of our medium resolution run is less than 0.01 radians in phase and $0.1 \%$ in amplitude for $t>1000 M$. At earlier times, the errors are somewhat larger and are dominated by residual junk radiation. Our uncertainty due to outer boundary effects is similar to that in Fig. 4: about 0.02 radians in phase and half a percent in amplitude. Boundary effects are most prominent during the ringdown.

\section{Extrapolation of waveforms to infinity}

Our numerical simulations cover only a finite spacetime volume, as shown in Fig. 1, so it is necessary to extract our numerical waveforms at a finite distance from the source. However, gravitational-wave detectors measure waveforms as seen by an observer infinitely far from the source. Accordingly, after extracting waveforms at multiple finite radii, we extrapolate these waveforms to infinite radius using a procedure similar to that described in [9]. This extrapolation procedure is intended to remove not only near-field effects that are absent at infinity, but also gauge effects that can be caused by the time dependence of the lapse function or the nonoptimal choice of tetrad for computing $\Psi_{4}$.

The extraction procedure described in Sec. III A yields a set of waveforms $\Psi_{4}^{22}(t, r)$, with each waveform extracted at a different radius. To extrapolate to infinite radius we must compare waveforms at different radii, but these waveforms must be offset in time by the light-travel time between adjacent radii. To account for this time shift, for each extraction radius we compute $\Psi_{4}^{22}(u, r)$, where $u$ is the retarded time at that radius. Assuming for simplicity that the background spacetime is nearly Schwarzschild, we compute the retarded time $u$ using

$$
u \equiv t_{s}-r^{*},
$$

where $t_{s}$ is some approximation of Schwarzschild time, and the tortoise-coordinate radius [90] is

$$
r^{*}=r_{\text {areal }}+2 M_{\mathrm{ADM}} \ln \left(\frac{r_{\text {areal }}}{2 M_{\mathrm{ADM}}}-1\right) .
$$

Here $M_{\mathrm{ADM}}$ is the ADM mass of the initial data, and $r_{\text {areal }}=\sqrt{A / 4 \pi}$, where $A$ is the measured (time-dependent) area of the extraction sphere. If we were to choose $t_{s}$ to be simply the coordinate time $t$, then the retarded time coordinate $u$ would fail to be null, largely because the lapse function in our simulation is time dependent and differs from the Schwarzschild value. We attempt to account for this by assuming that our background spacetime coordinates are Schwarzschild, but with $g_{t t}$ replaced by $-N_{\text {avg }}^{2}$, where $N_{\text {avg }}$ is the (time-dependent) average value of the lapse function measured on the extraction sphere. Under these assumptions, it can be shown that the one-form

$$
\frac{N_{\text {avg }}}{\sqrt{1-2 M_{\mathrm{ADM}} / r_{\text {areal }}}} d t-d r^{*}
$$

is null, so we equate this one-form with $d u$ and thus define

$$
t_{s}=\int_{0}^{t} \frac{N_{\mathrm{avg}}}{\sqrt{1-2 M_{\mathrm{ADM}} / r_{\text {areal }}}} d t .
$$

We show below (cf. Fig. 9) that choosing Eq. (42) instead of $t_{s}=t$ significantly increases the accuracy of our extrapolation procedure during merger and ringdown.

Having computed the retarded time at each extraction radius, we now consider the extracted waveforms as functions of retarded time $u$ and extraction radius $r_{\text {areal }}$, i.e. $\Psi_{4}^{22}\left(u, r_{\text {areal }}\right)$. At each value of $u$, we have the phase and amplitude of $\Psi_{4}^{22}$ at several extraction radii $r_{\text {areal }}$. Therefore at each value of $u$, we fit phase and amplitude separately to a polynomial in $1 / r_{\text {areal }}$ :

$$
\begin{aligned}
& \phi\left(u, r_{\text {areal }}\right)=\phi_{(0)}(u)+\sum_{k=1}^{n} \frac{\phi_{(k)}(u)}{r_{\text {areal }}^{k}}, \\
& r_{\text {areal }} A(u, r)=A_{(0)}(u)+\sum_{k=1}^{n} \frac{A_{(k)}(u)}{r_{\text {areal }}^{k}} .
\end{aligned}
$$

The phase and amplitude of the desired asymptotic waveform are thus given by the leading-order term of the appropriate polynomial, as a function of retarded time:

$$
\begin{gathered}
\phi(u)=\phi_{(0)}(u), \\
r_{\text {areal }} A(u)=A_{(0)}(u) .
\end{gathered}
$$

Figure 6 shows phase and amplitude differences between extrapolated waveforms that are computed using different values of polynomial order $n$ in Eqs. (43) and (44). For the extrapolation we use waveforms extracted at radii $75 M, 85 M, 100 M, 110 M, 130 M, 140 M, 150 M$, $160 M, 170 M, 180 M, 190 M, 200 M, 210 M$, and $225 M$. From Fig. 6 it is clear that increasing $n$ increases the accuracy of the extrapolation in smooth regions, but also amplifies any noise present in the waveform. Our preferred choice, $n=3$, gives a phase error of 0.005 radians and a relative amplitude error of 0.003 during most of the inspiral, and a phase error of 0.01 radians and a relative amplitude error of 0.01 in the ringdown. The junk radiation epoch $t_{s}-r^{*} \lesssim 1000 M$ has moderately larger errors than the ringdown. If we were to choose instead $n=4$, we would gain higher accuracy in the smooth regions at the expense of increased noise in the junk radiation epoch and slightly larger errors during the merger and ringdown.

Figure 7 is the same as the top panel of Fig. 6, except zoomed to late times. Note that during merger and ringdown, the extrapolation procedure does not converge with increasing extrapolation order $n$ : the phase differences are slightly larger for larger $n$. This lack of convergence sug- 


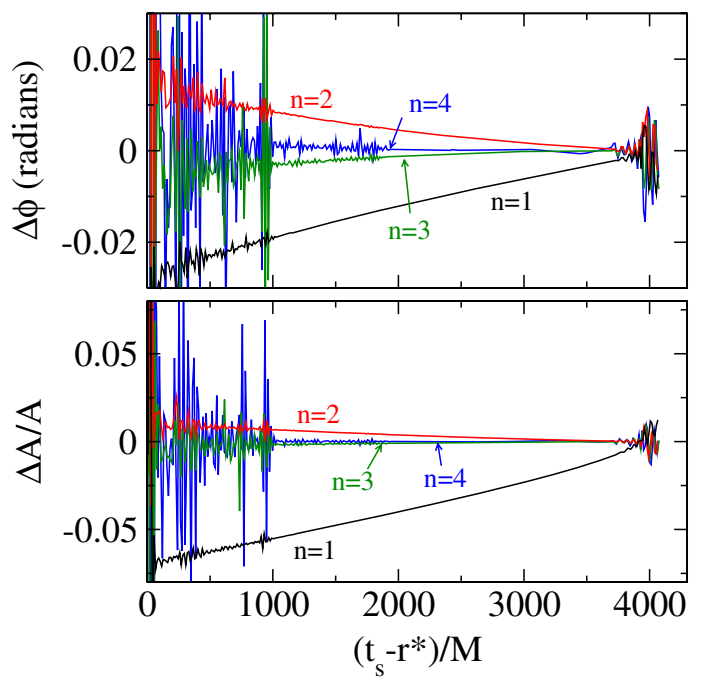

FIG. 6 (color online). Convergence of extrapolation to infinity for extrapolation of order $n$. For each $n$, plotted is the extrapolated waveform from run 30c1/N6 using order $n+1$ minus the extrapolated waveform using order $n$. The top panel shows phase differences, the bottom panel shows amplitude differences. No shifting in time or phase has been done for this comparison. Increasing $n$ increases accuracy in smooth regions but also amplifies noise.

gests that the nonextrapolated numerical waveform contains some small contamination that does not obey the fitting formulas, Eqs. (43) and (44). Figure 8 shows the $n=$ 1 and $n=2$ convergence curves from Fig. 7, but computed for two different numerical resolutions, 30c1/N5 and 30c1/ N6. The N5 and N6 lines are very close to each other in this figure, indicating that the lack of convergence with extrapolation order $n$ is not dominated by insufficient numerical resolution. We suspect that the main contribution is instead due to gauge effects. Such gauge effects might be reduced by improving the gauge conditions in the numerical simulation or by adopting more sophisticated wave extraction and extrapolation algorithms that better compensate for dynamically varying gauge fields.

Indeed, we have already made a first attempt at correcting for a time-dependent lapse function by using $t_{s}$ from Eq. (42) to compute the retarded time. Figure 9 illustrates

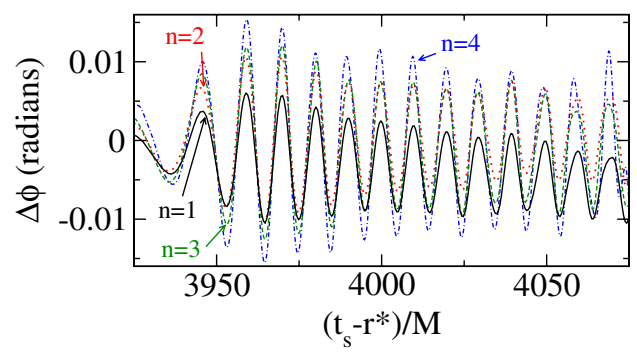

FIG. 7 (color online). Late-time phase convergence of extrapolation to infinity. Same as the top panel of Fig. 6, except zoomed to late times. The peak amplitude of the waveform occurs at $t_{s}-$ $r^{*}=3954 M$.

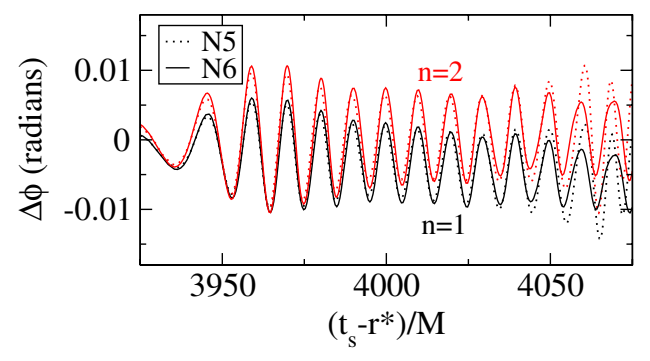

FIG. 8 (color online). Effect of numerical resolution on extrapolation to infinity. The solid curves are identical to the " $n=1$ " and " $n=2$ " curves from Fig. 7. The dotted curves are the same quantities computed using the lower resolution run $30 \mathrm{c} 1 / \mathrm{N} 5$.

the importance of this correction. Figures 7 and 9 differ only in the choice of $t_{s}$ used to compute the retarded time: In Fig. 7, $t_{s}$ is obtained from Eq. (42), and in Fig. 9, $t_{s}$ is simply the coordinate time $t$. Using the naive choice $t_{s}=t$ clearly results in much larger phase differences that diverge with increasing $n$ and grow in time.

In Fig. 10 we examine the difference between extrapolated waveforms and waveforms that have been extracted at a finite radius. We compare our preferred waveform, $30 \mathrm{c} 1 / \mathrm{N} 6$ extrapolated to infinity using $n=3$, versus nonextrapolated waveforms and versus extrapolated waveforms with different values of $n$. Because the extrapolated and nonextrapolated waveforms differ by overall time and phase offsets which are irrelevant for many purposes, each waveform in Fig. 10 has been shifted in time and phase so as to best match with the $n=3$ extrapolated waveform. This best match is determined by a simple least-squares procedure: we minimize the function

$f\left(t_{0}, \phi_{0}\right)=\sum_{i}\left(A_{1}\left(t_{i}\right) e^{i \phi_{1}\left(t_{i}\right)}-A_{2}\left(t_{i}+t_{0}\right) e^{i\left(\phi_{2}\left(t_{i}+t_{0}\right)+\phi_{0}\right)}\right)^{2}$,

by varying $t_{0}$ and $\phi_{0}$. Here $A_{1}, \phi_{1}, A_{2}$, and $\phi_{2}$ are the amplitudes and phases of the two waveforms being matched, and the sum goes over all times $t_{i}$ at which waveform 1 is sampled.

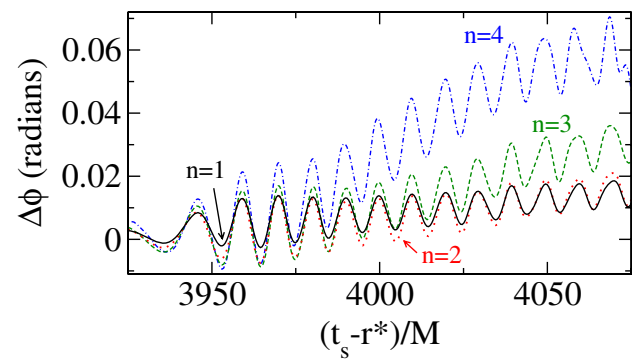

FIG. 9 (color online). Effect of $t_{s}$ on extrapolation to infinity. Same as Fig. 7, except the quantity $t_{s}$ that appears in the retarded time, Eq. (39), is chosen to be coordinate time $t$ rather than the integral in Eq. (42). Note the difference in vertical scale between this figure and Fig. 7. 


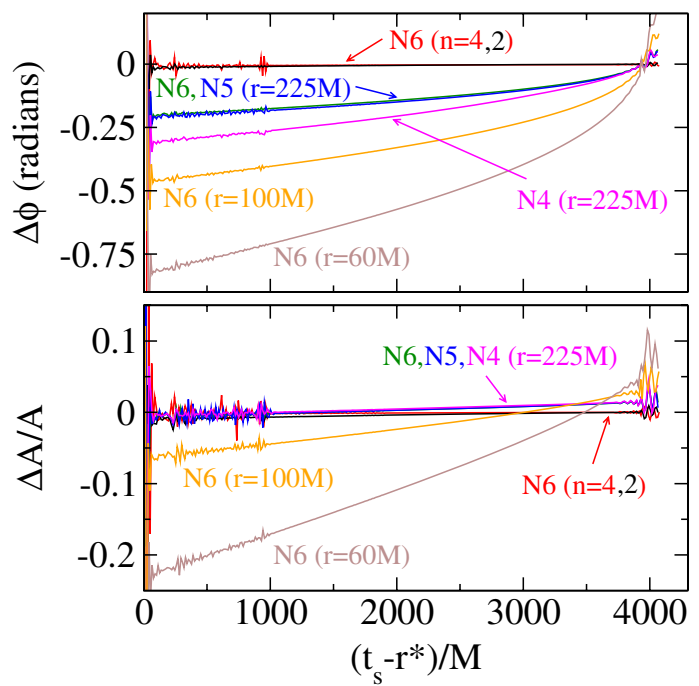

FIG. 10 (color online). Comparison of extrapolated and nonextrapolated waveforms. Plotted are differences between selected waveforms and the 30c1/N6 waveform extrapolated to infinity using $n=3$. Each selected waveform is labeled by the numerical resolution (N4, N5, or N6), and either the extraction radius (for nonextrapolated waveforms) or the extrapolation order (for extrapolated waveforms). Each waveform has been shifted in time and phase so as to minimize the least-squares difference from the N6, $n=3$ waveform. The top panel shows phase differences, the bottom panel shows amplitude differences. Differences between extrapolated and nonextrapolated waveforms are much larger than differences between different extrapolation orders. Phase differences between resolutions N5 and N6, and amplitude differences between all three resolutions, are indistinguishable on the plot.
We find from Fig. 10 that extrapolation to infinity has a large effect on the phase of the final waveform and a much smaller effect on the amplitude, when comparing to data extracted at our outermost extraction radius, $r=225 \mathrm{M}$. The $r=225 M$ waveforms have an accumulated phase difference of 0.2 radians relative to the extrapolated waveform, much larger than the difference between different extrapolation orders or different numerical resolutions. For extraction at smaller radii, the differences are larger still, the $r=60 M$ waveform having a phase difference of 0.8 radians and amplitude difference of $20 \%$ compared to the extrapolated waveform. We find that the phase differences between extrapolated and nonextrapolated waveforms scale quite accurately like $1 / r$, and the amplitude differences scale roughly like $1 / r^{2.5}$, where $r$ is the extraction radius. These scalings seem to be related to near-field effects, for which one expects scalings like $1 / r$ in phase and $1 / r^{2}$ in amplitude [86].

Figure 11 presents the final waveform after extrapolation to infinite radius. There are 33 gravitational-wave cycles before the maximum of $\left|\Psi_{4}\right|$. The simulation is further able to resolve ten gravitational-wave cycles during ringdown, during which the amplitude $\left|\Psi_{4}\right|$ drops by 4 orders of magnitude.

\section{DISCUSSION}

We have presented the first spectral computation of a binary black hole inspiral, merger, and ringdown, and we have extracted accurate gravitational waveforms from our simulation. A key ingredient in handling the merger phase
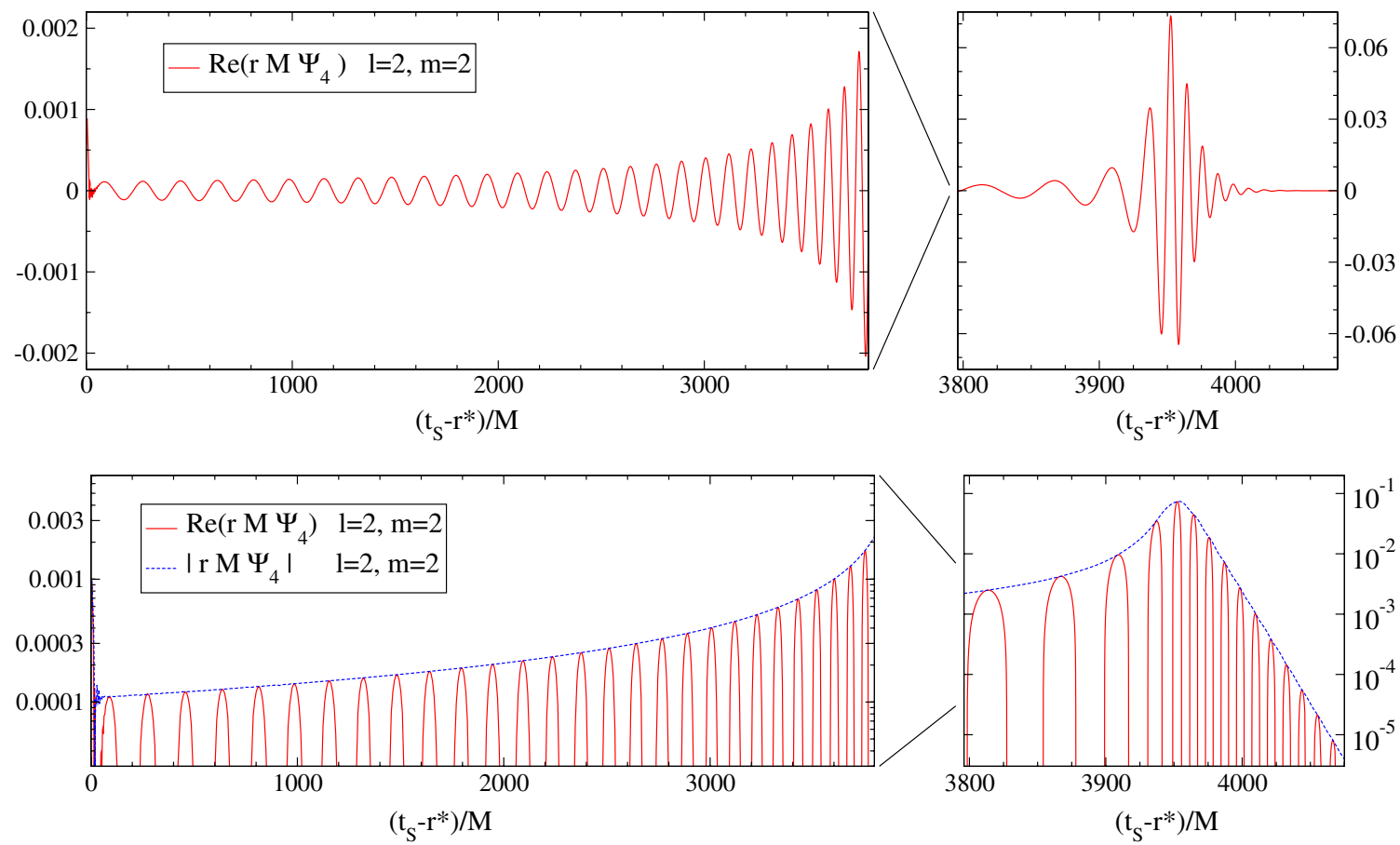

FIG. 11 (color online). Final waveform, extrapolated to infinity. The top panels show the real part of $\Psi_{4}^{22}$ with a linear $y$ axis, the bottom panels with a logarithmic $y$ axis. The right panels show an enlargement of merger and ringdown. 
is a choice of gauge that causes the individual holes to expand in coordinate size. This eliminates the coordinate singularities that prevented our earlier simulations from continuing through merger. The largest downside to the gauge used here is that the success of the method depends sensitively on some of the gauge parameters, namely $\sigma_{1}$ and $\sigma_{2}$ in Eq. (8), and $\xi_{1}$ and $\xi_{3}$ in Eqs. (6) and (7). If these parameters are chosen poorly, the characteristic fields at the excision boundaries fail to be purely outgoing (i.e. into the holes) at some instant in time, causing the code to terminate due to lack of a proper boundary condition at an excision boundary. An alternative approach to gauge conditions for the generalized harmonic system [77] is in progress, and promises to be more robust.

We compute the spin of the final black hole with three distinct diagnostics, one based on approximate rotational Killing vectors, the others based on the minimum and maximum of the scalar curvature of the apparent horizon $\left(\chi_{\mathrm{AKV}}, \chi_{\mathrm{SC}}^{\min }\right.$, and $\chi_{\mathrm{SC}}^{\max }$ in the language of Appendices A and $\mathrm{B}$ of [55]). We find that these diagnostics agree to an exquisite degree. Since these diagnostics coincide exactly for a Kerr black hole, this suggests that the final state is indeed a Kerr black hole. The uncertainty of the final spin quoted in Sec. II E is due to numerical truncation error (i.e. differences between resolutions 30c1/N5 and 30c1/N6), rather than due to differences between spin diagnostics, and we find $S_{f} / M_{f}^{2}=0.68646 \pm 0.00004$, and $M_{f}=$ $(0.95162 \pm 0.00002) M$.

The physical waveform at infinity produced by any numerical relativity code should of course be independent of the coordinates used during the simulation. However, in practice it is difficult to remove coordinate effects from the waveform for several reasons. First, waveforms are typically extracted on coordinate spheres (not geometric spheres) of finite radius as functions of coordinate time (which may not agree with proper time at infinity). Second, the extracted waveform on a given sphere is typically expanded in spin-weighted spherical harmonics ${ }_{s} Y_{\ell m}(\theta, \phi)$ using the $\theta$ and $\phi$ coordinates from the simulation rather than some geometrically defined $\theta$ and $\phi$ coordinates. Finally, standard formulas equating $\Psi_{4}$ with the asymptotic radiation field assume that $\Psi_{4}$ is computed at infinity. Such gauge ambiguities can be significant for the accuracy of waveforms from numerical simulations [87-89]. Indeed, if we choose a deliberately "bad" gauge just after merger by omitting the factor $e^{-r^{12} / \sigma_{3}^{2}}$ in the function $f(x, t)$ [cf. Equation (30)], we find that the lapse function oscillates in time even at large distances, and that the resulting waveform extracted at a finite radius differs by more than a radian in phase from the waveform presented here. We defer further discussion of gauge effects on the waveform to a future paper.
We have also shown that extrapolation of waveforms to infinity is crucial: waveforms extracted at a finite radius differ (particularly in phase) from waveforms extrapolated to infinity by far more than the numerical errors, as shown in Fig. 10. Although it is likely that the need for extrapolation may be somewhat reduced by more sophisticated algorithms for wave extraction at finite radius, it appears that most of the difference between waveforms that have and have not been extrapolated to infinity is due to physics (in the form of near-zone effects) rather than to gauge and tetrad ambiguities [86].

We are currently extending our methods to binary black holes with unequal masses and nontrivial spins. Inspiral simulations for these more generic systems have already been computed by our code; it remains to be seen whether mergers of more generic black hole systems can be simulated with the methods described here, or whether alternative gauge conditions, such as those described in Ref. [77], will be necessary.

It would be interesting to compare the waveforms presented here with those from other groups computing binary black hole mergers, particularly since other groups use different numerical methods, different formulations of the equations, and different gauge conditions than our group. Several such comparisons are presently under way.

Waveforms are available at http://www.black-holes.org/ Waveforms.html.

\section{ACKNOWLEDGMENTS}

We thank Luisa Buchman, Luis Lehner, Frans Pretorius, Bela Szilágyi, Saul Teukolsky, and Kip Thorne for helpful comments, Fan Zhang for help with the extrapolation code, and Geoffrey Lovelace and Rob Owen for providing the diagnostics used to measure the final spin. We are especially grateful to Lee Lindblom for numerous suggestions, ideas, and discussions that significantly contributed to the success of the methods described here. This work was supported in part by grants from the Sherman Fairchild Foundation to Caltech and Cornell, and from the Brinson Foundation to Caltech; by NSF Grants No. PHY-0601459, No. PHY-0652995, No. DMS-0553302, and NASA Grant No. NNG05GG52G at Caltech; by NSF Grants No. PHY0652952, No. DMS-0553677, No. PHY-0652929, and NASA Grant No. NNG05GG51G at Cornell. We thank NASA/JPL for providing computing facilities that contributed to this work. Some of the simulations discussed here were produced with LIGO Laboratory computing facilities. LIGO was constructed by the California Institute of Technology and Massachusetts Institute of Technology with funding from the National Science Foundation and operates under Cooperative Agreement No. PHY-0107417. 
[1] F. Pretorius, Phys. Rev. Lett. 95, 121101 (2005).

[2] M. Campanelli, C. O. Lousto, P. Marronetti, and Y. Zlochower, Phys. Rev. Lett. 96, 111101 (2006).

[3] J. G. Baker, J. Centrella, D.-I. Choi, M. Koppitz, and J. van Meter, Phys. Rev. Lett. 96, 111102 (2006).

[4] A. Buonanno, G. B. Cook, and F. Pretorius, Phys. Rev. D 75, 124018 (2007).

[5] J. G. Baker, J.R. van Meter, S.T. McWilliams, J. Centrella, and B. J. Kelly, Phys. Rev. Lett. 99, 181101 (2007).

[6] Y. Pan, A. Buonanno, J. G. Baker, J. Centrella, B. J. Kelly, S. T. McWilliams, F. Pretorius, and J. R. van Meter, Phys. Rev. D 77, 024014 (2008).

[7] A. Buonanno, Y. Pan, J. G. Baker, J. Centrella, B. J. Kelly, S. T. McWilliams, and J. R. van Meter, Phys. Rev. D 76, 104049 (2007).

[8] M. Hannam, S. Husa, J. A. González, U. Sperhake, and B. Brügmann, Phys. Rev. D 77, 044020 (2008).

[9] M. Boyle, D. A. Brown, L. E. Kidder, A. H. Mroué, H. P. Pfeiffer, M. A. Scheel, G. B. Cook, and S. A. Teukolsky, Phys. Rev. D 76, 124038 (2007).

[10] A. Gopakumar, M. Hannam, S. Husa, and B. Brügmann, Phys. Rev. D 78, 064026 (2008).

[11] M. Hannam, S. Husa, B. Brügmann, and A. Gopakumar, Phys. Rev. D 78, 104007 (2008).

[12] M. Boyle, A. Buonanno, L. E. Kidder, A. H. Mroué, Y. Pan, H. P. Pfeiffer, and M. A. Scheel, Phys. Rev. D 78, 104020 (2008).

[13] A. H. Mroué, L. E. Kidder, and S. A. Teukolsky, Phys. Rev. D 78, 044004 (2008).

[14] I. Hinder, F. Herrmann, P. Laguna, and D. Shoemaker, http://www.arxiv.org/abs/0806.1037.

[15] M. Campanelli, C. O. Lousto, and Y. Zlochower, Phys. Rev. D 74, 041501(R) (2006).

[16] M. Campanelli, C. O. Lousto, and Y. Zlochower, Phys. Rev. D 74, 084023 (2006).

[17] M. Campanelli, C. O. Lousto, Y. Zlochower, B. Krishnan, and D. Merritt, Phys. Rev. D 75, 064030 (2007).

[18] F. Herrmann, I. Hinder, D. M. Shoemaker, P. Laguna, and R. A. Matzner, Phys. Rev. D 76, 084032 (2007).

[19] P. Marronetti, W. Tichy, B. Brügmann, J. González, and U. Sperhake, Phys. Rev. D 77, 064010 (2008).

[20] E. Berti, V. Cardoso, J. A. Gonzalez, U. Sperhake, and B. Brügmann, Classical Quantum Gravity 25, 114035 (2008).

[21] M. Campanelli, Classical Quantum Gravity 22, S387 (2005).

[22] F. Herrmann, I. Hinder, D. Shoemaker, and P. Laguna, Classical Quantum Gravity 24, S33 (2007).

[23] J. G. Baker, J. Centrella, D.-I. Choi, M. Koppitz, J. R. van Meter, and M. C. Miller, Astrophys. J. 653, L93 (2006).

[24] J. A. Gonzalez, U. Sperhake, B. Brügmann, M. Hannam, and S. Husa, Phys. Rev. Lett. 98, 091101 (2007).

[25] M. Campanelli, C. O. Lousto, Y. Zlochower, and D. Merritt, Phys. Rev. Lett. 98, 231102 (2007).

[26] J.A. Gonzalez, M.D. Hannam, U. Sperhake, B. Brügmann, and S. Husa, Phys. Rev. Lett. 98, 231101 (2007).

[27] B. Brügmann, J. A. González, M. Hannam, S. Husa, and U. Sperhake, Phys. Rev. D 77, 124047 (2008).

[28] F. Herrmann, I. Hinder, D. Shoemaker, P. Laguna, and R. A. Matzner, Astrophys. J. 661, 430 (2007).
[29] D.-I. Choi, B. J. Kelly, W.D. Boggs, J. G. Baker, J. Centrella, and J. van Meter, Phys. Rev. D 76, 104026 (2007).

[30] J. G. Baker, W. D. Boggs, J. Centrella, B. J. Kelly, S. T. McWilliams, M. C. Miller, and J. R. van Meter, Astrophys. J. 668, 1140 (2007).

[31] W. Tichy and P. Marronetti, Phys. Rev. D 76, 061502(R) (2007).

[32] J.D. Schnittman, A. Buonanno, J. R. van Meter, J. G. Baker, W. D. Boggs, J. Centrella, B. J. Kelly, and S. T. McWilliams, Phys. Rev. D 77, 044031 (2008).

[33] M. Campanelli, C. O. Lousto, Y. Zlochower, and D. Merritt, Astrophys. J. Lett. 659, L5 (2007).

[34] M. Koppitz, D. Pollney, C. Reisswig, L. Rezzolla, J. Thornburg, P. Diener, and E. Schnetter, Phys. Rev. Lett. 99, 041102 (2007).

[35] S. H. Miller and R. Matzner, arXiv:0807.3028.

[36] J. G. Baker, W. D. Boggs, J. Centrella, B. J. Kelly, S. T. McWilliams, M. C. Miller, and J. R. van Meter, Astrophys. J. 682, L29 (2008).

[37] J. Healy, F. Herrman, I. Hinder, D. M. Schoemaker, P. Laguna, and R. A. Matzner, arXiv:0807.3292 [Phys. Rev. Lett. (to be published)].

[38] L. S. Finn, Phys. Rev. D 46, 5236 (1992).

[39] P. Ajith et al., Classical Quantum Gravity 24, S689 (2007).

[40] T. Damour and A. Nagar, Phys. Rev. D 77, 024043 (2008).

[41] T. Damour, A. Nagar, M. Hannam, S. Husa, and B. Brügmann, Phys. Rev. D 78, 044039 (2008).

[42] L. Lindblom, B. J. Owen, and D. A. Brown, http://www.arxiv.org/abs/0809.3844 [Phys. Rev. D (to be published)].

[43] S. Bonazzola, J. Frieben, E. Gourgoulhon, and J.-A. Marck, in ICOSAHOM'95, Proceedings of the Third International Conference on Spectral and High Order Methods, edited by A. V. Ilin and L. R. Scott (Houston Journal of Mathematics, Houston, 1996), pp. 3-19.

[44] S. Bonazzola, E. Gourgoulhon, and J.-A. Marck, Phys. Rev. Lett. 82, 892 (1999).

[45] P. Grandclément, S. Bonazzola, E. Gourgoulhon, and J.-A. Marck, J. Comput. Phys. 170, 231 (2001).

[46] E. Gourgoulhon, P. Grandclément, and S. Bonazzola, Phys. Rev. D 65, 044020 (2002).

[47] P. Grandclément, E. Gourgoulhon, and S. Bonazzola, Phys. Rev. D 65, 044021 (2002).

[48] H. P. Pfeiffer, G. B. Cook, and S. A. Teukolsky, Phys. Rev. D 66, 024047 (2002).

[49] H. P. Pfeiffer, L.E. Kidder, M. A. Scheel, and S. A. Teukolsky, Comput. Phys. Commun. 152, 253 (2003).

[50] G. B. Cook and H. P. Pfeiffer, Phys. Rev. D 70, 104016 (2004).

[51] M. Ansorg, B. Brügmann, and W. Tichy, Phys. Rev. D 70, 064011 (2004).

[52] M. Ansorg, Phys. Rev. D 72, 024018 (2005).

[53] M. Caudill, G. B. Cook, J. D. Grigsby, and H. P. Pfeiffer, Phys. Rev. D 74, 064011 (2006).

[54] P. Grandclément, Phys. Rev. D 74, 124002 (2006).

[55] G. Lovelace, R. Owen, H. P. Pfeiffer, and T. Chu, Phys. Rev. D 78, 084017 (2008).

[56] F. Foucart, L.E. Kidder, H. P. Pfeiffer, and S. A. Teukolsky, Phys. Rev. D 77, 124051 (2008).

[57] M. A. Scheel, H. P. Pfeiffer, L. Lindblom, L. E. Kidder, O. Rinne, and S. A. Teukolsky, Phys. Rev. D 74, 104006 
(2006).

[58] J. W. York, Phys. Rev. Lett. 82, 1350 (1999).

[59] H. P. Pfeiffer and J. W. York, Phys. Rev. D 67, 044022 (2003).

[60] G. B. Cook, Phys. Rev. D 65, 084003 (2002).

[61] H. P. Pfeiffer, D. A. Brown, L. E. Kidder, L. Lindblom, G. Lovelace, and M. A. Scheel, Classical Quantum Gravity 24, S59 (2007).

[62] L. Lindblom, M. A. Scheel, L. E. Kidder, R. Owen, and O. Rinne, Classical Quantum Gravity 23, S447 (2006).

[63] H. Friedrich, Commun. Math. Phys. 100, 525 (1985).

[64] D. Garfinkle, Phys. Rev. D 65, 044029 (2002).

[65] F. Pretorius, Classical Quantum Gravity 22, 425 (2005).

[66] O. Rinne, Classical Quantum Gravity 23, 6275 (2006).

[67] O. Rinne, L. Lindblom, and M. A. Scheel, Classical Quantum Gravity 24, 4053 (2007).

[68] J. M. Stewart, Classical Quantum Gravity 15, 2865 (1998).

[69] H. Friedrich and G. Nagy, Commun. Math. Phys. 201, 619 (1999).

[70] J. M. Bardeen and L. T. Buchman, Phys. Rev. D 65, 064037 (2002).

[71] B. Szilágyi, B. Schmidt, and J. Winicour, Phys. Rev. D 65, 064015 (2002).

[72] G. Calabrese, J. Pullin, O. Reula, O. Sarbach, and M. Tiglio, Commun. Math. Phys. 240, 377 (2003).

[73] B. Szilágyi and J. Winicour, Phys. Rev. D 68, 041501(R) (2003).

[74] L. E. Kidder, L. Lindblom, M. A. Scheel, L. T. Buchman, and H. P. Pfeiffer, Phys. Rev. D 71, 064020 (2005).

[75] L. T. Buchman and O.C. A. Sarbach, Classical Quantum Gravity 23, 6709 (2006).

[76] L. T. Buchman and O.C. A. Sarbach, Classical Quantum
Gravity 24, S307 (2007).

[77] L. Lindblom, K. D. Matthews, O. Rinne, and M. A. Scheel, Phys. Rev. D 77, 084001 (2008).

[78] F. Pretorius, Classical Quantum Gravity 23, S529 (2006).

[79] B. Szilágyi, D. Pollney, L. Rezzolla, J. Thornburg, and J. Winicour, Classical Quantum Gravity 24, S275 (2007).

[80] M. Holst, L. Lindblom, R. Owen, H. P. Pfeiffer, M. A. Scheel, and L. E. Kidder, Phys. Rev. D 70, 084017 (2004).

[81] G. B. Cook and B. F. Whiting, Phys. Rev. D 76, 041501(R) (2007).

[82] R. Owen, Ph. D. thesis, California Institute of Technology, 2007, http://resolver.caltech.edu/CaltechETD:etd05252007-143511.

[83] M. Campanelli, C. O. Lousto, and Y. Zlochower, Phys. Rev. D 73, 061501(R) (2006).

[84] J. G. Baker, J. Centrella, D.-I. Choi, M. Koppitz, and J. van Meter, Phys. Rev. D 73, 104002 (2006).

[85] B. Brügmann, J. A. González, M. Hannam, S. Husa, U. Sperhake, and W. Tichy, Phys. Rev. D 77, 024027 (2008).

[86] M. Boyle and A. H. Mroué (unpublished).

[87] A. Nerozzi, M. Bruni, L. M. Burko, and V. Re, in Proceedings of the Albert Einstein Century International Conference, Paris, France, 2005, pp. 702-707 [AIP Conf. Proc. 861, 702 (2006)].

[88] E. Pazos, E. N. Dorband, A. Nagar, C. Palenzuela, E. Schnetter, and M. Tiglio, Classical Quantum Gravity 24, S341 (2007).

[89] L. Lehner and O. M. Moreschi, Phys. Rev. D 76, 124040 (2007).

[90] D. R. Fiske, J. G. Baker, J. R. van Meter, D.-I. Choi, and J. M. Centrella, Phys. Rev. D 71, 104036 (2005). 